\title{
From centennial to millennial oscillation of the thermohaline circulation
}

\author{
Florian Sévellec ${ }^{1, *}$, Thierry Huck $^{2}$ and Alain Colin de Verdière ${ }^{2}$ \\ ${ }^{1}$ Department of Geology and Geophysics, Yale University, 210 Whitney Avenue, PO Box 208109, New Haven, \\ CT 06520-8109, USA. \\ ${ }^{2}$ Laboratoire de Physique des Océans (UMR 6523 CNRS IFREMER IRD UBO), Brest, France \\ *: Corresponding author : Florian Sévellec, email address : florian.sevellec@yale.edu
}

\begin{abstract}
:
The freshwater flux intensity is used as the main control parameter to destabilize the ThermoHaline Circulation (THC): as it increases, the classical bifurcation scenario, thermal stable steady state, millennial oscillation and haline stable steady state are reproduced in a 2D zonally-averaged ocean model under mixed boundary conditions. Both bifurcations arise through global bifurcations leading to an infinite period of the limit cycle. Here we highlight a centennial oscillation important for the instability of the thermal phase of the millennial oscillation and which could be considered as a precursor to the collapse of the THC. It is suggested that the existence or the absence of this centennial oscillation leads to dramatic changes in the collapse time scale of the THC going from several thousand years to only a few decades. This bifurcation scenario is shown to be sensitive to the value of the dissipation.
\end{abstract}




\section{Introduction}

The Holocene climate is characterized by its remarkable stability during the last ten thousand years compared to the climate of the last 250,000 years (Dansgaard et al., 1993). This relative stability has been detected in the analyses of ice core data which show a strong variability of the last 123,000 years on a millennial time scale (GRIP Members, 1993; Alley, 2000; NGRIP Members, 2004). Strong episodes, called Dansgaard-Oeschger events (DOE), make up a series of climate shifts with a cyclicity of about $1470 \pm 500$ years (Bond et al., 1997; Grootes and Stuiver, 1997; Bond et al., 1999). They can be analyzed in two phases: a rapid global warming of 5 to $10^{\circ} \mathrm{C}$ occurring in few decades followed by slow cooling during several centuries. It has been conjectured very early that these DOE are related to variations of the thermohaline circulation (Broecker et al., 1990), the two phases being respectively associated with strong and weak ThermoHaline Circulation (THC hereafter). However, Wunsch (2000) has questioned the very existence of the millennial oscillation and has suggested instead that the millennial variability might be due to the aliasing of the seasonal cycle in the observations. Schulz (2002) emphasized the nonstationary character of the times series of oxygen isotopes as only a few DOE contribute to the spectral peak of $1470 \mathrm{yr}$ making the aliasing hypothesis unnecessary.

Independently of this discussion about the observations, the existence of multiple equilibria of the THC was found by Stommel (1961). This study illustrated the ability of the THC to explain the two phases of the DOE. Later, a series of study (Thual and McWilliams, 1992; Quon and Ghil, 1992; Cessi and Young, 1992) shows that the 2D thermohaline circulation was also able to generate these multiple equilibria solutions. More recently, this theory was confirmed using ocean general circulation model in forced (Rahmstorf et al., 2005) or coupled with an atmosphere configuration (Manabe and Stouffer, 1988; 1999), as well as proxy data (McManus et al., 2004). However Cessi and Young (1992) have shown that, in the limit of dominant vertical diffusion where temperature and salinity are vertically uniform, the steady states exhibited in the $2 \mathrm{D}$ thermohaline circula- 
tion were inexorably stable and thus do not fully explain the DOE.

The millennial oscillations have been reproduced in ocean models under mixed boundary conditions, i.e. restoring on temperature and flux on salinity (Weaver and Sarachik, 1991; Weaver et al., 1993; Winton and Sarachik, 1993; Winton, 1993; 1995). Recent works in 2D zonally-averaged and box models have shown, through the construction of bifurcation diagrams, the existence of a window of millennial oscillations for intermediate values of surface freshwater flux. Furthermore the period of this oscillation was shown to depend on the distance to the bifurcation (Colin de Verdière et al., 2006; CBS06 in what follows). Colin de Verdière (2007) also provides a conceptual dynamical system to generate these millennial oscillations, which highlights the importance of convection to restart the phase of the strong THC as in Cessi (1996).

Since the freshwater flux is known as an essential parameter to set the steady or variable character of ocean model circulations, modifications of the water cycle (an expected consequence of global warming) may be seen as threats to the present steady state of the actual circulation. Josey and Marsh (2005) show that the sea surface salinity has changed since mid-1970s due to the increase of the precipitation in the North Atlantic subpolar gyre. During the last decade, modifications of salinity have also appeared at depth (Curry et al., 2003; Curry and Mauritzen, 2005). Given this freshwater change and the existence of millennial oscillations, we raise the question of the stability of the present THC and wonder if this change might be able to trigger glacial events.

We have performed a sensitivity analysis to better characterize the occurrence and the nature of bifurcations as we vary the freshwater flux. CBS06 have found that two bifurcations, both defined as global bifurcation (an infinitely small modification in parameter leads to the appearance of a finite cycle, Dijkstra, 2000) separate three regimes: thermally dominant, oscillatory and salinity dominant. In this study, we show that the characteristic of the first bifurcation is strongly linked to the existence of a centennial oscillation. This has strong implications for the period of the millennial oscillation and the evolution of the 
ocean circulation during the oscillation.

The 2D zonally-averaged model is described in Section 2. and this is followed by a description of the different regimes occurring through the change of the freshwater flux intensity. The nature of the two bifurcations are identified in Section 3. and is shown to depend on the value of the dissipation (here a linear Rayleigh friction). A discussion of the results is given in Section $4 .$.

\section{The ocean model}

\section{a. Dynamical system equations}

The ocean model is a latitude-depth 2D ocean model similar to that used by CBS06 but written in Cartesian coordinates (Sévellec et al., 2006; SHB06 in what follows):

$$
\begin{gathered}
-\rho_{0}^{-1} \partial_{y} P-\varepsilon v=0, \\
-\partial_{z} P-\rho g=0, \\
\partial_{y} v+\partial_{z} w=0 .
\end{gathered}
$$

where $y$ is the latitude, $z$ is the vertical coordinate, $P$ is the pressure, $\rho\left(\rho_{0}\right)$ is the (reference) density, $(v, w)$ is the velocity, and $g$ the gravity acceleration. The choice of the linear friction coefficient $\varepsilon=1.45 \times 10^{-4} \mathrm{~s}^{-1}$ leads to a realistic overturning $\simeq 15 \mathrm{~Sv}$ for a typical North Atlantic thermohaline stratification and width. The dynamics correspond to a very frictional system with a momentum balance between the meridional pressure gradient and the linear friction, as in the Stommel (1961) box model. To some extent, this approximation, compared to the classical 2D primitive equations, aims to represents an $\mathrm{ad}$ hoc parameterization of the rotation.

A linearized equation of state for the seawater is used:

$$
\rho=\rho_{0}\left[1-\alpha\left(T-T_{0}\right)+\beta\left(S-S_{0}\right)\right]
$$


where $\alpha$ is the thermal expansion coefficient, $T\left(T_{0}\right)$ is the (reference) temperature, $\beta$ is the haline contraction coefficient, and $S\left(S_{0}\right)$ is the (reference) salinity.

Only the thermodynamic equations are prognostic:

$$
\begin{aligned}
& \partial_{t} T=-J(\psi, T)+K_{H} \partial_{y}^{2} T+K_{V} \partial_{z}^{2} T+\mathcal{F}_{\mathcal{T}}+\mathcal{C}_{\mathcal{T}} \\
& \partial_{t} S=-J(\psi, S)+K_{H} \partial_{y}^{2} S+K_{V} \partial_{z}^{2} S+\mathcal{F}_{\mathcal{S}}+\mathcal{C}_{\mathcal{S}}
\end{aligned}
$$

where $J$ is the Jacobian operator, $\psi$ is the overturning streamfunction defined as $w=\partial_{y} \psi$ and $v=-\partial_{z} \psi, K_{H}\left(K_{V}\right)$ is the horizontal (vertical) eddy diffusivity and $\mathcal{C}$ the convection. The convection instantaneously mixes the temperature and the salinity downward (conserving the heat and salt content) until $\partial_{z} \rho<=0$. Surface boundary conditions for temperature and salinity differ ("mixed boundary conditions") to take into account the existence of a feedback between the sea surface temperature (SST) and surface heat flux, but its absence in the case of salinity. The surface forcing is then expressed as:

$$
\begin{aligned}
\mathcal{F}_{T} & =\tau_{T}^{-1}\left[T^{*}(y)-\operatorname{SST}(y)\right] \\
\mathcal{F}_{S} & =\frac{S_{0}}{h} F_{W}
\end{aligned}
$$

appearing only in the uppermost model level of thickness $h=50 \mathrm{~m} ; \tau_{T}$ is the restoring time scale for temperature, and the restoring surface temperature and the freshwater flux read:

$$
\begin{aligned}
T^{*}(y) & =T_{0}^{*}[1+\cos (\pi y / \tilde{y})], \\
F_{W}(y) & =-F_{0} \sin \left[2 \pi\left(y-y_{0}\right) /\left(y_{1}-y_{0}\right)\right],
\end{aligned}
$$

where latitude $y$ varies between southern and northern boundaries at $y=y_{0}$ and $y=y_{1}$, and $\tilde{y}$ is the latitudinal extent parameter of the restoring surface temperature.

These equations are solved using a finite difference formulation (see Table 1 for the 
model parameters values), on a uniform latitudinal grid but non-uniform vertical grid (15 levels of thickness varying from $50 \mathrm{~m}$ at the surface to $550 \mathrm{~m}$ at the bottom). No-normalflow conditions are used on all boundaries, resulting in zero streamfunction, and zero flux conditions are applied to temperature and salinity except at the surface.

\section{b. Multiple equilibria and millennial oscillation}

Time integrations of the 2D model for different intensities of the freshwater flux reveal two different stable steady states (Fig. 1), as in Stommel (1961). Although the subject of the present work focuses on the transitions from such steady states, we describe them briefly here for completeness. For low values of the freshwater flux the steady state corresponds to a thermally-driven stratification intensified in the 500 first meters (Fig. 2). This temperature dominated stratification induces a northward (southward) surface (deep) mass flux, with downwelling associated with convection in the northern region and a global upwelling elsewhere.

For large freshwater flux the steady state corresponds to a stratification intensified in the first 1000 meters (Fig. 2). The stratification is now salinity dominated and induces a southward surface mass flux, a downwelling in the south and a northward upwelling recirculation. Two secondary cells localized along the southern and northern boundaries are characterized by northward surface mass fluxes, due to the inversion of the freshwater flux gradient, hence of the salinity gradient, at the highest and lowest latitude.

These two steady states are respectively named thermal and haline steady state; their parameters range of existence are respectively named the thermal and haline regimes.

Between these two regimes, time integrations of the 2D model show the periodic variability (Fig. 1) found by CBS06. This variability appears as a nonlinear oscillation on millennial time scale (Fig. 3), and the corresponding parameters range will be called the millennial oscillation regime.

The first slow phase (the thermal phase), corresponding to the poleward surface circulation occurring after the strong flush in the intensity of the circulation, has a circulation 
and a stratification close to those of the thermal steady state (Fig. 3). The rapid interphase after the thermal phase is due to a positive feedback which grows up toward the haline local equilibrium. Depending on the parameter regime (notably on the intensity of the freshwater flux), this feedback is an unstable salinity dominated centennial oscillation (SHB06) or the fully-studied positive salinity feedback (Marotzke, 1996). As it will be discussed later in this study, the control of the instability of the thermal phase by one or the other process is fundamental to set the timescale of the thermal phase.

The second slow phase (the haline phase) is characterized by an increase of the basinaveraged temperature and a weak equatorward surface circulation. The circulation is close to that of the haline steady state with a shallower circulation (Fig. 3). The deep ocean is warmed by both the advection and diffusion from the tropical surface layer. This warming slowly reduces the stratification and finally reactivates the convection in the north by a static instability (CBS06 and Colin de Verdière, 2007). This reactivation of the convection in the north triggers the outbreak of the thermal phase. Cold water is injected from the surface, poleward pressure forces build up and the thermal circulation occurs. This circulation pushes back the warm deep water towards the equator and reinforce the poleward pressure forces. This leads to a circulation occurring as a flush which overshoots the nearby thermal steady state (Fig. 3, upper right). There is a strong heat loss as the SST is restored to the atmospheric temperature (Fig. 3, upper left). By so doing the thermal horizontal gradient weakens and the overturning slows down.

However, the convection is not as crucial in our model as in idealized 2 degreesof-freedom model (such as Cessi, 1996; Colin de Verdière, 2007) for the existence of millennial oscillation. Actually, time integrations of our model setting $\mathcal{C}=0$ at each time step do provide millennial oscillation with mainly the same characteristics. In this latter case, the reactivation of the thermal phase is due to the weakening of the stratification until a large-scale instability of the meridional pressure gradient stops the haline phase. Actually our 2D model, with much larger degrees of freedom $\left(n_{y} \times n_{z}-1=849\right)$, allows 
instability of the haline phase through different process than just convective one.

\section{Characterization of the bifurcations}

A distinctive characteristic of the millennial oscillation is the evolution of its period as a function of the freshwater flux intensity (Fig. 5). Before discussing the properties of the millennial oscillation we should first define the two bifurcations surrounding the millennial oscillation regime. The thermal bifurcation is defined as the bifurcation between the regime of the thermal steady state and of the millennial oscillations. The haline bifurcation is defined as the bifurcation between the regime of haline steady states and of millennial oscillations. For both bifurcations the period of the millennial oscillation increases as the distance to the bifurcation decreases and its limit value goes to infinity. Such bifurcations are both called global bifurcation, however the two bifurcations have different behavior. For the thermal bifurcation the period increases to infinite as a -1.7 power law of the bifurcation distance (Fig. 5). For the haline bifurcation, the period follows a logarithm law (Fig. 5) which is characteristic of a homoclinic bifurcation (homoclinic orbit to a simple saddle, Strogatz, 1994). Although these two laws emerge from the dynamical system proposed by Colin de Verdière (2007), CBS06 did not observe the bifurcation with a period going to the infinite for the first bifurcation, a difference which prompted the present study.

How can we understand the bifurcation with a period going to infinity?, i.e. the increase of the time spent during a periodic trajectory near a bifurcation? The infinite period is due to trajectory that slows down because of the remnant attractor of what could be called a ghost of a steady state, an effect called a bottleneck. In other words, closer to the bifurcation, the higher is the attraction of the ghost of the steady state and the slower is the trajectory around the ghost of the steady state. At the bifurcation, the trajectory cuts the steady state and the slow-down becomes infinite, hence also the period. 


\section{a. Thermal bifurcation}

To better understand this millennial cycle and more particularly the two bifurcations, we analyse phase portraits of the basin-averaged temperature. This phase portrait is defined as the second time derivative as a function of the first time derivative and is performed for different values of the freshwater intensity around the two bifurcations. These plots allow us to see how the cycle is modified to reach a stable steady state.

For the thermal bifurcation, several time integrations are performed for different intensities of the freshwater flux (Fig. 6). The existence of a high frequency (centennial time scale, Fig. 4, right) during both thermal stable steady state and thermal phase is revealed. The mean temperature and the circulation maximum show that the increase of the period is controlled by the time spent during the thermal phase (Fig. 7).

The phase portrait reveals that the millennial cycle trajectory is always present in our range of parameters, but it intersects the zero value $\left(d_{t}^{2} T_{\text {mean }}=d_{t} T_{\text {mean }}=0\right.$, i.e. no evolution of the dynamical system) for the low values of the freshwater flux intensity, meaning that there is a stable fixed point (first two columns of Fig. 6). This fixed point evolves as a stable spiral fixed point, meaning that the attraction of the stable steady state occurs through an oscillatory mode. For higher value of the freshwater flux, the stable steady state disappears through a Hopf bifurcation: the stable spiral fixed point becomes an unstable fixed point but is not associated with a limit cycle of centennial period (as fully described by Sévellec et al., 2006). This bifurcation of the thermal steady state defines the bifurcation with a period going to infinity. At this point, the trajectories correspond to the millennial oscillations with centennial oscillation during the thermal phase. This resulting homoclinic orbit shows that the attraction is purely exponential whereas the expansion is oscillatory (Fig. 6). These characteristics have been defined as the Shilnikov-type phenomena of a homoclinic bifurcation (Shilnikov et al., 1998; 2001) and leads to a 3 degrees of freedom oscillation.

The oscillatory expansion shows the importance of the centennial variability to desta- 
bilize the thermal phase and set the period of the oscillation. On the one hand, the period of the millennial cycle close to the bifurcation is controlled by the time spent in the thermal phase, which is controlled by the instability of the centennial cycle (Fig. 7). On the other hand, the millennial cycle period does not change sufficiently far from the bifurcation, and does not exhibit anymore higher frequencies in the thermal phase. As expected from SHB06, the increase of the freshwater flux intensity leads to a more and more unstable thermal phase (Fig. 7), controlling to some extent the decrease of the millennial cycle period: $\mathcal{P}_{m} \propto \lambda_{c}^{-\alpha}$ (where $\mathcal{P}_{m}$ is the millennial cycle period, $\lambda_{c}$ the growth rate of the centennial cycle instability, and $\alpha$ a power law coefficient).

Given these last results, we conclude that the bottleneck effect, part of the infinite period is due to the time spent near the ghost of the thermal steady state. At the Hopf bifurcation the expansion of the steady state is zero $\left(\lambda_{c}=0\right)$, and thus the millennial cycle period is infinite $\left(\mathcal{P}_{m}=\infty\right)$. This is a typical way to obtain infinite period at the bifurcation.

The decrease of the time spend in the thermal phase with the increase of the freshwater flux reaches a saturation value. At this point, as described in SHB06, the instability is no longer controlled by the centennial oscillation but by the positive salinity feedback (Marotzke, 1996). In this regime, the instability time scale is quicker than the period of the centennial oscillation (set by the mean advection along the overturning). This explains why we lose track of the centennial frequency in the thermal phase for high value of the freshwater flux intensity.

\section{b. Haline bifurcation}

For the haline bifurcation, the time evolution of the mean temperature and of the circulation intensity reveals that the increase of the period near the second bifurcation is due to the increase of the time spent in the haline phase (Fig. 8). When the freshwater intensity increases, the millennial cycle is modified and in the neighborhood of the zero point $\left(\partial_{t}^{2} T_{\text {mean }}=\partial_{t} T_{\text {mean }}=0\right)$ a centennial stable limit cycle appears during the haline phase. This variability corresponds to a salinity dominated perturbation (Fig. 4, right) passively 
advected by the mean flow. This bifurcation defines the homoclinic bifurcation of the millennial oscillation into a haline limit cycle. As for the thermal bifurcation, we have determined that the homoclinic orbit shows a purely exponential attraction but an oscillatory expansion (Shilnikov et al., 1998; 2001) and leads to a 3 degrees of freedom oscillation (as for the thermal bifurcation). Then, if the freshwater flux intensity continues to increase, the amplitude of the limit cycle decreases until it becomes a stable steady point. The amplitude decreases following a square root law (Fig. 9, bottom), and the period does not change ( $\sim 140 \mathrm{yr})$, as expected for a supercritical Hopf bifurcation (Strogatz, 1994).

\section{c. Influence of the linear friction coefficient}

In order to test the importance of the previous results, we have performed further integrations using different values for the linear friction coefficient (Table 1), since this is a rather poorly known parameter in 2D models. This coefficient summarizes the parameterization of the 3D rotating dynamics in a zonally-averaged latitude-depth approximation and has a potentially important influence on the ocean adjustment to thermohaline changes. Using it as a control parameter allows us to test the relevance of the afore-mentioned bifurcations structure of such millennial oscillations and to test the governing mechanisms.

The first result is that the linear friction coefficient has an impact on both thermal and haline steady states. In both cases the intensity of the circulation is weaker for larger linear friction coefficient (Fig. 10).

The second result is the impact of the linear friction coefficient on the millennial oscillations. Although these oscillations exist under different values of the linear friction coefficient, their period changes (Fig. 10). Most importantly, the thermal bifurcation with an infinite period behavior disappears for high values of the linear friction coefficient. This regime explains why the CBS06 study carried out with higher dissipation did not observe centennial oscillations.

As discussed in section 3.a., the period of the millennial oscillation around the thermal bifurcation is controlled by the growth of the centennial oscillation. This means that 
understanding the disappearance of the centennial oscillation through change of the linear friction coefficient can explain the disappearance of the bifurcation with an infinite period behavior of the millennial cycle.

SHB06 show that the existence of centennial oscillations (see their equation 34) is controlled by the ratio between the response time scale of the THC to the salinity forcing and the THC time scale $\left(\tau_{\mathrm{O}} / \tau_{\mathrm{SF}}<1\right)$. The former is a typical time scale of the circulation such as a measure of the renewal time scale based on the transport and the volume of the basin. The latter is a typical time scale of the freshwater forcing: a measure of how fast the atmospheric freshwater is injected into the oceanic mixed layer. As they show in their study the first time scale is proportional to the square root of the linear friction coefficient intensity and to the inverse of the square root of the freshwater forcing intensity $\left(\tau_{\mathrm{SF}} \propto \sqrt{\varepsilon / F_{0}}\right.$ where $\varepsilon$ could be related to $1 / k$ - the inverse of the proportional factor between the overturning and the buoyancy torque - in their study). The second time scale is proportional to the linear friction coefficient $\left(\tau_{\mathrm{O}} \propto \varepsilon\right)$. Note that the renewal time scale of the thermal steady state of our 2D model evolves also following the linear friction coefficient $\left(\psi_{\max } \propto 1 / \varepsilon\right.$, Fig. 10). It follows that the condition of the existence of centennial oscillation is governed by the square root of the freshwater forcing multiplied by the linear friction coefficient $\left(\tau_{\mathrm{O}} / \tau_{\mathrm{SF}} \propto \sqrt{F_{0} \varepsilon}\right)$. This last result demonstrates that the existence of the centennial oscillation, and thus the bifurcation with an infinite period behavior, is not only controlled by the freshwater intensity but also by the friction coefficient.

Therefore it is not surprising that the increase of the linear friction coefficient, with a low freshwater flux intensity, exhibits the same kind of bifurcation (with an infinite period behavior) as the increase of the freshwater flux intensity, with a low linear friction coefficient. Actually, as for the freshwater flux intensity, a low value of the linear friction coefficient leads to a stable spiral steady state during the thermal phase, and it is the stability of this spiral steady state that controls the period of the millennial oscillation. Nevertheless for higher values, when the centennial oscillatory direction of the spiral 
steady state disappears, the thermal bifurcation with an infinite period behavior does no longer appear.

As previously discussed in section 3.a. and in SHB06, this modification in the thermal bifurcation corresponds to a control of the thermal phase instability by the centennial oscillation or by the positive salinity feedback. This result rationalizes the difference between our study and CBS06.

The last result is the impact of the use of horizontal Laplacian diffusion $\left(\nu \partial_{y y} v\right)$ for viscosity instead of the linear friction in (1a). In this context, the millennial oscillations regime exists only for unrealistic value of freshwater flux intensity: $F_{0}>3 \mathrm{~m} \mathrm{yr}^{-1}$ (with $\left.\nu=3 \times 10^{7} \mathrm{~m}^{2} \mathrm{~s}^{-1}\right)$. This result could explain why some previous studies report only stable steady states (Thual and McWilliams, 1992; Quon and Ghil, 1992; Cessi and Young, 1992).

\section{Discussion}

The stability of the ThermoHaline Circulation (THC) has been discussed here in a 2D zonally-averaged model. A particular attention was made to characterize the precursor of the collapse of the THC due to an increase of the freshwater flux. The link between the possible collapse of the THC and the millennial oscillation was also investigated through sensitivity experiments of the influence of freshwater flux on circulation.

In this work we have taken a particular attention to characterize the bifurcations between the stable thermal and haline steady state regimes and the oscillation regime. Colin de Verdière et al. (2006) had found that these bifurcations were global bifurcations, the oscillations appearing through the bifurcation at finite amplitude. Consequently, small changes in the freshwater flux led to dramatic changes in the circulation dynamics. Such bifurcations have been rationalized with the dynamical system of Colin de Verdière (2007; its Figs. 9 and 10 should be compared to our Figs. 5 and 10).

In the present experiments, the existence of global bifurcations for the thermal bifur- 
cation is directly related to the existence of the centennial oscillation. As explained by Sévellec et al. (2006), the existence of the centennial oscillation is linked to the low value of friction coefficient and freshwater flux. Here the values corresponding to the realistic circulation intensity lead to this centennial oscillation. This centennial oscillation occurs as the destabilization of the thermal phase and is an important part of the millennial oscillation sketch (consistent with the Shilnikov-type bifurcation). We thus interpret that, for the present circulation under realistic regime, the centennial oscillation is a precursor of the collapse of the THC.

Our study on the sensitivity of the friction coefficient highlights the importance of the ratio between the THC time scale and the response time scale of the overturning to the salinity forcing. In our idealized model, this ratio sets the thermal bifurcation type from an infinite period bifurcation to a finite one. Then, in this study, the collapse of the circulation could occur in thousand of year or in less than one hundred years. Thus if the THC is sufficiently slow compared to the ocean surface feedback, this will lead to a dramatic change in few decades. Given the range of uncertainty of the collapse time scale in such a simple model (which takes into account a small subset of climate feedbacks), we should pay a particular attention to the position of the present climate in the proper bifurcation diagram, if we want to predict any of its change.

Following Sévellec et al. (2006), this centennial oscillation becomes strongly damped in two hemispheres when the freshwater flux is symmetric with respect to the equator. This leads to the question of the appearance of the millennial oscillation in a two hemispheres configuration. However, even if stable, internal mode of the thermohaline circulation could be excited under stochastic forcing (te Raa and Dijkstra, 2003). In this context, Mikolajewicz and Maier-Reimer (1990) and Mysak et al. (1993) have shown that the centennial oscillation in two hemispheres could be triggered by atmospheric noise. Mysak et al. (1993) have also shown that in presence of this atmospheric noise the millennial oscillation could be sustained in two hemispheres. In these cases, although this millennial 
oscillation is an internal oceanic mode, its appearance is strongly linked to the existence of an external source of energy (coming from atmospheric synoptic processes).

We can speculate that the present climate is related to what we have previously called the stable thermal steady state. However we have no certitude that the present circulation corresponds to a stable steady state or to an extremely long thermal phase of a millennial oscillation. If the present circulation corresponds to a thermal steady state, an increase of the freshwater flux intensity (due to global warming and sufficiently slow to consider the ocean circulation as adjusted) will result in a global bifurcation: that is, the appearance of a millennial cycle with glacial event (haline phase). In the other case, if the present circulation corresponds to a thermal phase of a millennial oscillation, two sub-cases appear. In the first sub-case, the present climate is far enough from the second bifurcation (the haline bifurcation), and an increase of the freshwater flux intensity will result in a decrease of the ratio between the times spent in the thermal phase and the haline phase. This corresponds to a dramatic increase in the duration of glacial events and the time spent in the present circulation could be reduced from several thousand years to only few hundred years. In the second sub-case, the present climate is close to the haline bifurcation: an increase of the freshwater flux intensity will lead to a switch in a stable glacial steady state (the stable haline steady state).

Our model and configuration allowed us to conduct sensitivity experiments in a broad parameter range. We have thus been able to check the robustness and describe the bifurcation occurring through changes in the freshwater flux intensity. In particular, we have shown the importance of centennial oscillation to set the time scale of the circulation shutdown. Although our configuration has overlooked many aspects of the ocean circulation which could have a role in the collapse of the THC (the Antarctic Circumpolar region, the wind forcing, the three-dimensional geometry, the geostrophic turbulence or the air-sea interaction for instance), this study and the existence of a strong signature of the centennial variability in the North Atlantic (Stocker and Mysak, 1992) highlight that the role of the 
centennial variability should be addressed in future work on prediction of THC collapse. 


\section{REFERENCES}

Alley, R. B. 2000. Ice-core evidence of abrupt climate changes. Proc. Nat. Acad. Sci. U.S.A., 97, 1331-1334.

Bond, G., W. Showers, M. Cheseby, R. Lottu, P. Almasi, P. deMenocal, P. Priore, H. Cullen, I. Hajdas, and G. Bonani. 1997. A pervasive millenial-scale cycle in north atlantic holocene and glacial climates. Science, 278, 1257-1266.

Bond, G., W. Showers, M. Elliot, M. Evans, R. Lotti, I. Hajdas, g. Bonani, and S. Johnson. 1999. The north atlantic's 1-2 kyr climate rhythm: relation to heinrich events, dansgaard-oeschger events, and the little ice age. Geophys. Monogr. Ser., 112, 35-58.

Broecker, W. S., G. Bond, M. Klas, G. Bonani, and W. Wolfli. 1990. A salt oscillator in the glacial atlantic? 1. the concept. Paleoceanography, 5, 469-477.

Cessi, P. 1996. Convective adjustment and thermohaline excitability. J. Phys. Oceanogr., $26,481-491$.

Cessi, P. and W. R. Young. 1992. Multiple equilibria in two-dimensional thermohaline circulation. J. Fluid Mech., 241, 291-309.

Colin de Verdière, A. 2007. A simple model of millenial oscillations of the thermohaline circulation. J. Phys. Oceanogr., 37, 1142-1155.

Colin de Verdière, A., M. Ben Jelloul, and F. Sévellec. 2006. Bifurcation structure of thermohaline oscillation. J. Climate, 19, 5777-5795.

Curry, R. and C. Mauritzen. 2005. Dilution of the northern north atlantic ocean in recent decades. Science, 308, 1772-1774. 
Curry, R., B. Dickson, and I. Yashayaev. 2003. A change in freshwater balance of the atlantic ocean over the past four decades. Nature, 426, 826-829.

Dansgaard, W., S. J. Johnsen, H. B. clausen, D. Dahl-Jensen, N. S. Gundestrup, C. U. Hammer, C. S. Hvldberg, J. P. Steffensen, A. E. Sveinbjörnsdóttir, J. Jouzel, and G. Bond. 1993. Evidence for general instability of past climate from a 250-kyr icecore record. Nature, 364, 218-220.

Dijkstra, H. A. 2000. Nonlinear Physical Oceanography. Kluwer Academic Publishers.

GRIP Members. 1993. Climate instability during the last interglacial period recorded in the grip ice core. Nature, 364, 203-207.

Grootes, P. M. and M. Stuiver. 1997. Oxygen 18/16 variability in greenland snow and ice with 10-3 to 105-year time resolution. J. Geophys. Res., 102, 26455-26470.

Josey, S. A. and R. Marsh. 2005. Surface freshwater flux variability and recent freshening of the north atlantic in the eastern subpolar gyre. J. Geophys. Res., 110, C05008.

Manabe, S. and R. J. Stouffer. 1988. Two stable equilibria of a coupled ocean-atmosphere model. J. Climate, 1, 841-866.

Manabe, S. and R. J. Stouffer. 1999. Are two modes of thermohaline circulation stable? Tellus, 51A, 400-411.

Marotzke, J. 1996. pages 333-378. Analysis of thermohaline feedbacks. In: Decadal climate variability. Dynamics and predictability, D. L. T. Anderson and J. Willebrand (eds.). NATO ASI Series, Series I, vol. 44.

McManus, J. F., R. Francois, J.-M. Gherardi, L. D. Keigwin, and S. Brown-Leger. 2004. Collapse and rapid resumption of atlantic meridional circulation linked to deglacial claimate changes. Nature, 428, 834-836. 
Mikolajewicz, U. and E. Maier-Reimer. 1990. Internal secular variability in an ocean general circulation model. Clim. Dyn., 4, 145-156.

Mysak, L. A., T. F. Stocker, and F. Huang. 1993. Century-scale variability in a randomly forced, two-dimensional thermohaline ocean circulation model. Clim. Dyn., 8, 103106.

NGRIP Members. 2004. High-resolution record of northern hemisphere climate extending into the last interglacial. Nature, 431, 147-151.

Quon, C. and M. Ghil. 1992. Multiple equilibria in thermosolutal convection due to salt-flux boundary conditions. J. Fluid Mech., 245, 449-483.

Rahmstorf, S., M. Crucifix, A. Ganopolski, H. Goosse, I. Kamenkovich, R. Knutti, G. Lohmann, R. Marsh, M. L. A., Z. Wang, and A. J. Weaver. 2005. Thermohaline circulation hysteresis: a model intercomparison. Geophys. Res. Lett., 32, L23605.

Schulz, M. 2002. On the 1470-year pacing of dansgaard-oeschger warm events. Paleoceanography, 17, doi:10.1029/2000PA000571.

Sévellec, F., T. Huck, and M. Ben Jelloul. 2006. On the mechanism of centennial thermohaline oscillations. J. Mar. Res., 64, 355-392.

Shilnikov, L. P., A. Shilnikov, D. Turaev, and L. Chua. 1998. Methods of Qualitative Theory in Nonlinear Dynamics: Part I. World Scientific Series on Nonlinear Science.

Shilnikov, L. P., A. Shilnikov, D. Turaev, and L. Chua. 2001. Methods of Qualitative Theory in Nonlinear Dynamics: Part II. World Scientific Series on Nonlinear Science.

Stocker, T. F. and L. A. Mysak. 1992. Climatic fluctuations on the century time scale: a review of high-resolution proxy data and possible mechanisms. Clim. Chan., 20, $227-250$. 
Stommel, H. 1961. Thermohaline convection with stable regimes flow. Tellus, 13, 224230.

Strogatz, S. H. 1994. Nonlinear dynamics and chaos with applications to physics, biology, chemistry and engineering. Advanced book program, Perseus book.

te Raa, L. A. and H. A. Dijkstra. 2003. Modes of internal thermohaline variability in a single-hemispheric ocean basin. J. Mar. Res., 61, 491-516.

Thual, O. and J. C. McWilliams. 1992. The catastrophe structure of thermohaline convection in a two-dimensional fluid model and a comparison with low-order box models. Geophys. Astrophys. Fluid Dynamics, 64, 67-95.

Weaver, A. J. and E. S. Sarachik. 1991. The role of mixed boundary conditions in numerical models of ocean's climate. J. Phys. Oceanogr., 21, 1470-1493.

Weaver, A. J., J. Marotzke, P. F. Cummins, and E. S. Sarachik. 1993. Stability and variability of the thermohaline circulation. J. Phys. Oceanogr., 23, 39-60.

Winton, M. 1993. pages 417-432. Deep decoupling oscillations of the oceanic thermohaline circulation. In: Ice in the climate system, W. R. Peltier, Springer Verlag (eds.). NATO ASI Series, Series I, vol. 12.

Winton, M. 1995. Energetics of deep-decoupling oscillations. J. Phys. Oceanogr., 25, $420-427$.

Winton, M. and E. S. Sarachik. 1993. Thermohaline oscillations induced by strong steady salinity forcing of ocean general circulation models. J. Phys. Oceanogr., 23, 1389-1410.

Wunsch, C. 2000. On sharp spectral lines in the climate record and the millenial peak. Paleoceanography, 15, 417-424. 


\section{List of Figures}

1 Bifurcation diagram for the 2D ocean model. For each experiment, the crosses denote either the stable steady-state value or the minimum and maximum values if it is an oscillation. The vertical dashed lines denote the bifurcation points (from left: two global bifurcations and the Hopf bifurcation). . . . . . . . . . . . . . . .

2 Left (right), thermal (haline) steady state characterized by a strong stratification of the temperature and salinity in the first 500 (1000) meters, the former (latter) one dominate the density stratification. The induced circulation is intensified at the surface with a northward (southward) mass transport and a downwelling in the north (south). The haline circulation is also characterized by two weak secondary cells, localized in the south and the north, both inducing northward surface flux. The solid, dashed and dotted lines correspond respectively to positive, negative and zero anomalies: contour interval is respectively $2^{\circ} \mathrm{C}, 0.5 \mathrm{psu}$ and $1 \mathrm{~Sv}$ from (left) 4 to $24^{\circ} \mathrm{C}, 35$ to $37 \mathrm{psu}$ and 0 to $15 \mathrm{~Sv}$; (right) 4 to $25^{\circ} \mathrm{C}, 29$ to $36 \mathrm{psu}$ and -10 to $3 \mathrm{~Sv}$.

3 Time integration of the 2D ocean model for a freshwater flux intensity of $105 \mathrm{~m} \mathrm{yr}^{-1}$. (top left) The basin-averaged temperature during an oscillation exhibits two phases: a rapid cooling, followed by a slow warming (denoted by the vertical solid lines and the letters $\mathrm{T}$ and $\mathrm{H}$ respectively). (top right) The maximum circulation exhibits the same two phases corresponding respectively to a positive and negative cell separated by a flush occurring after the rapid cooling. The dotted lines correspond to the two snapshots plotted. Left (right) snapshot during the cooling (warming) phase of the temperature, salinity and meridional overturning circulation are close to the thermal (haline) steady state: strong stratification of the 500 (1000) first meters and northward (southward) surface flux. The solid, dashed and dotted lines respectively correspond to positive, negative and zero anomalies: $\mathrm{CI}$ is respectively $2^{\circ} \mathrm{C}, 0.5 \mathrm{psu}$ and $1 \mathrm{~Sv}$ from (left) 4 to $24^{\circ} \mathrm{C}, 35$ to $37 \mathrm{psu}$ and 0 to $15 \mathrm{~Sv}$; (right) 4 to $25^{\circ} \mathrm{C}, 31$ to $37 \mathrm{psu}$ and -4 to $3 \mathrm{~Sv}$.

4 (left) Temperature, salinity (both in terms of density) and meridional overturning anomaly snapshots, of a phase and a quarter period later, of the oscillation occurring during the thermal bifurcation of the millennial oscillation. (right) As left for the limit cycle occurring close to the haline bifurcation. The period of this centennial oscillation are respectively 187 and $138 \mathrm{yr}$ for a freshwater flux intensity of 94 and $185 \mathrm{~m} \mathrm{yr}^{-1}$. The solid, dashed and dotted lines respectively correspond to positive, negative and zero anomalies: CI is respectively $5 \times 10^{-6}\left(5 \times 10^{-7}\right)$ and $0.5\left(10^{-2}\right) \mathrm{Sv}$. .

5 Millennial oscillation period as a function of the intensity of the freshwater flux: it becomes infinite near the two bifurcations, following an -0.17 power (logarithm) law between the regime of thermal (haline) steady state and of the millennial oscillation. . . . . . . . . . . . . . 
6 Time integration of the model for 6 different values of the freshwater flux around the first bifurcation. The maximum of the meridional overturning circulation (first row) and the mean temperature (second), show the appearance of the millennial oscillation and of higher frequency oscillation during the temperature steady state and phase. The phase portrait for the entire cycle (third row) and a blow-up around the zero value (fourth) show a stable spiral fixed point moving into an unstable spiral fixed points, then the trajectories follow the millennial stable cycle. The increasing freshwater flux increases the instability of the spiral fixed point. . . . . . . . . .

7 Millenial oscillations characteristics as a function of the freshwater flux amplitude: period (top left), time spent in the thermal (top right) and haline phase (bottom right) during a millennial oscillation, growth time scale of the thermal phase instability (bottom left). . . . . . . . . .

8 As in Fig. 6 for the second bifurcation. The diagrams show, trough the modification of the freshwater flux intensity, the appearance of an oscillation during the haline phase of the millennial cycle, then the appearance of a stable limit cycle which becomes weaker and weaker until it becomes a stable point (corresponding to the stable haline steady state). . . . . . .

9 (top) As in Fig. 1, around the haline bifurcation. The dashed line repre-
sents the mean value of the limit cycle. (bottom) Amplitude of the stable
limit cycle (+) as a function of the distance to the bifurcation, the square
root law (solid line) characterizes the presence of a supercritical Hopf bi-

9 (top) As in Fig. 1, around the haline bifurcation. The dashed line repre-
sents the mean value of the limit cycle. (bottom) Amplitude of the stable
limit cycle (+) as a function of the distance to the bifurcation, the square
root law (solid line) characterizes the presence of a supercritical Hopf bi-

9 (top) As in Fig. 1, around the haline bifurcation. The dashed line repre-
sents the mean value of the limit cycle. (bottom) Amplitude of the stable
limit cycle (+) as a function of the distance to the bifurcation, the square
root law (solid line) characterizes the presence of a supercritical Hopf bi-

9 (top) As in Fig. 1, around the haline bifurcation. The dashed line repre-
sents the mean value of the limit cycle. (bottom) Amplitude of the stable
limit cycle (+) as a function of the distance to the bifurcation, the square
root law (solid line) characterizes the presence of a supercritical Hopf bifurcation.

10 (right) Circulation intensity of the thermal and haline steady states through modification of the linear friction coefficient. (left) Millennial oscillation period as a function of freshwater flux intensity and linear friction coefficient. The first bifurcation behavior strongly depends of the linear friction coefficient: for the two series of experiments with the highest friction, the first bifurcation is no longer a bifurcation with an infinite period behavior. 


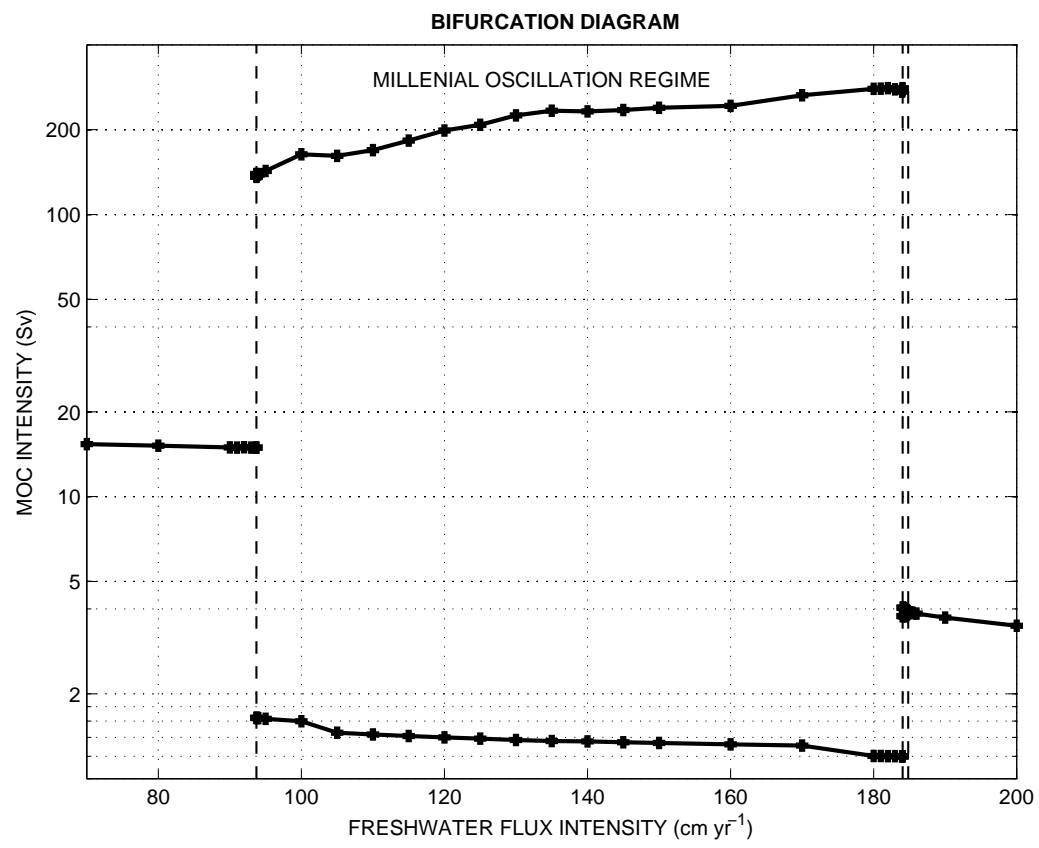

Figure 1: Bifurcation diagram for the 2D ocean model. For each experiment, the crosses denote either the stable steady-state value or the minimum and maximum values if it is an oscillation. The vertical dashed lines denote the bifurcation points (from left: two global bifurcations and the Hopf bifurcation). 

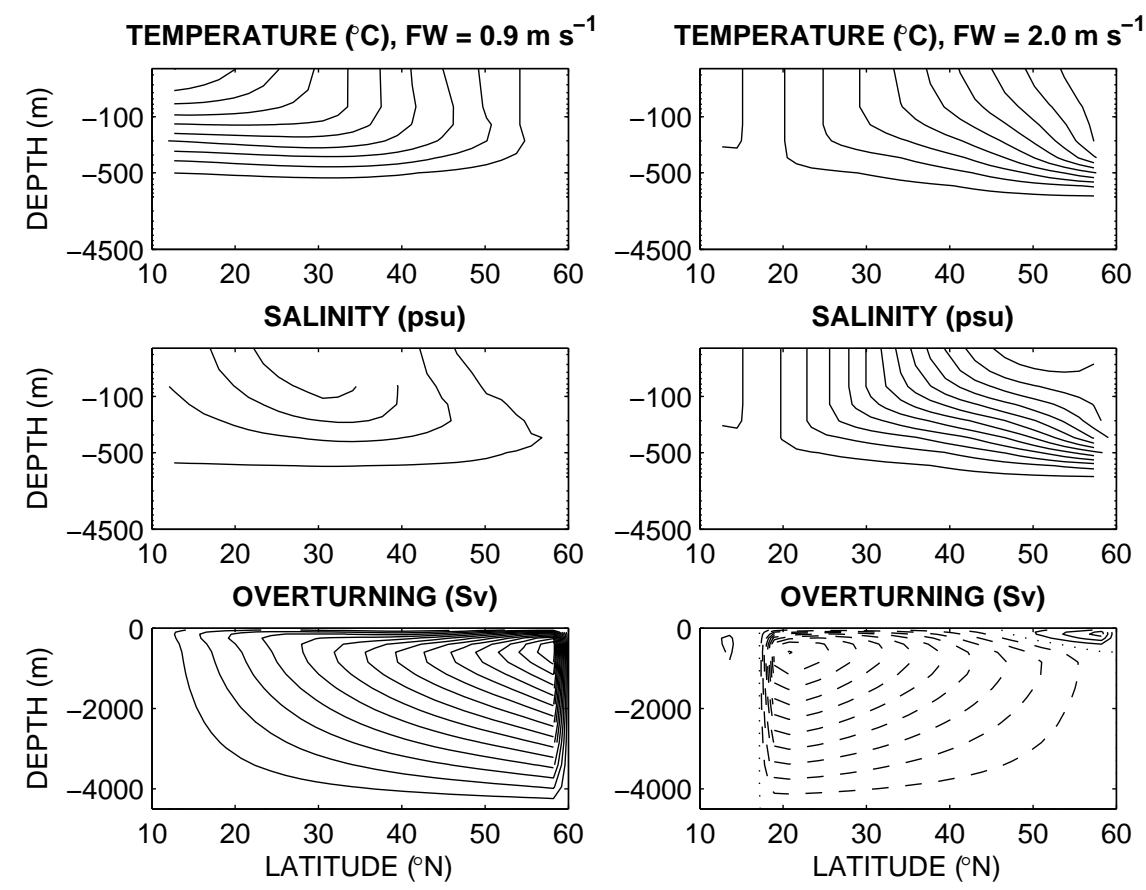

Figure 2: Left (right), thermal (haline) steady state characterized by a strong stratification of the temperature and salinity in the first 500 (1000) meters, the former (latter) one dominate the density stratification. The induced circulation is intensified at the surface with a northward (southward) mass transport and a downwelling in the north (south). The haline circulation is also characterized by two weak secondary cells, localized in the south and the north, both inducing northward surface flux. The solid, dashed and dotted lines correspond respectively to positive, negative and zero anomalies: contour interval is respectively $2^{\circ} \mathrm{C}, 0.5 \mathrm{psu}$ and $1 \mathrm{~Sv}$ from (left) 4 to $24^{\circ} \mathrm{C}, 35$ to $37 \mathrm{psu}$ and 0 to $15 \mathrm{~Sv}$; (right) 4 to $25^{\circ} \mathrm{C}, 29$ to 36 psu and -10 to $3 \mathrm{~Sv}$. 

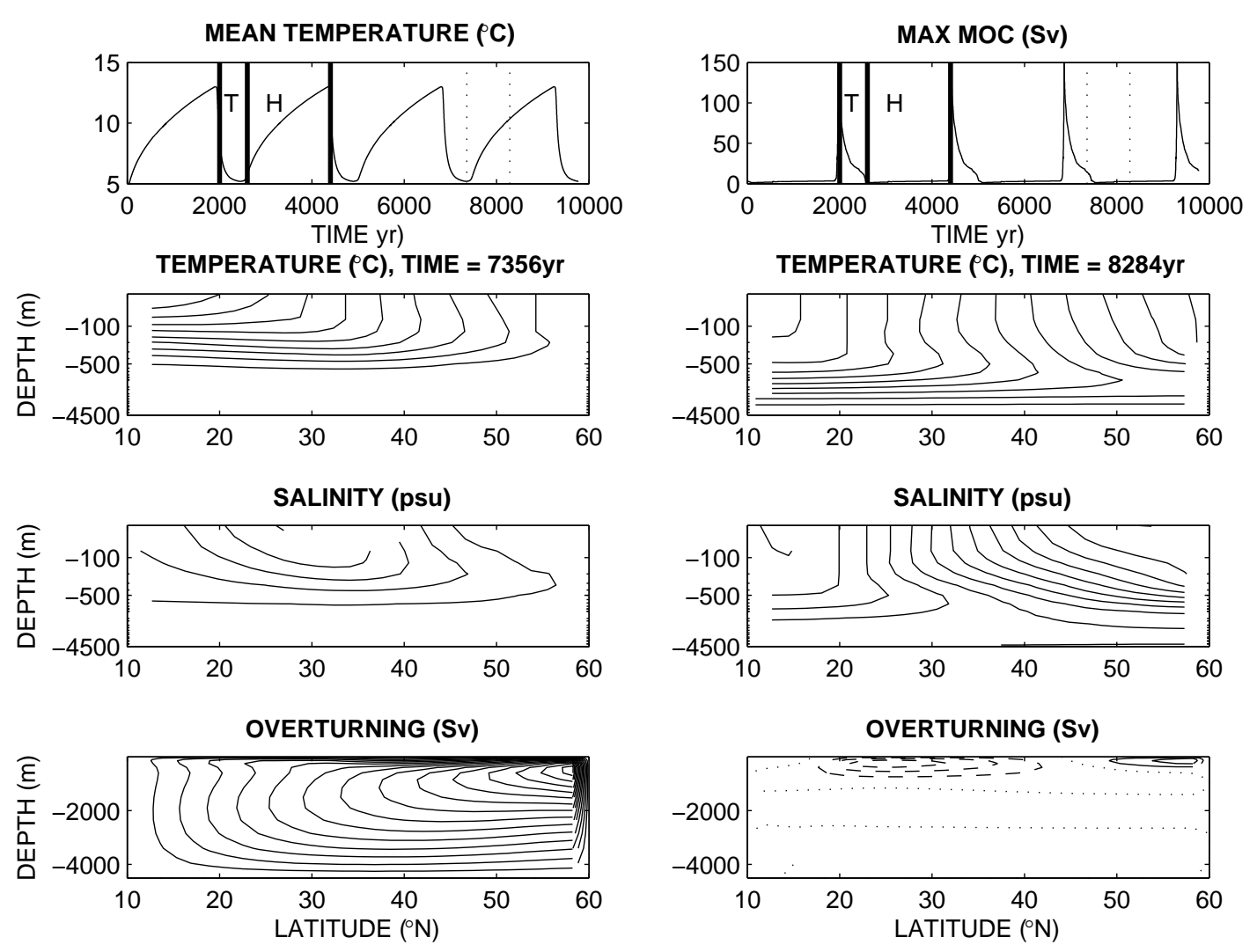

Figure 3: Time integration of the 2D ocean model for a freshwater flux intensity of $105 \mathrm{~m} \mathrm{yr}^{-1}$. (top left) The basin-averaged temperature during an oscillation exhibits two phases: a rapid cooling, followed by a slow warming (denoted by the vertical solid lines and the letters $\mathrm{T}$ and $\mathrm{H}$ respectively). (top right) The maximum circulation exhibits the same two phases corresponding respectively to a positive and negative cell separated by a flush occurring after the rapid cooling. The dotted lines correspond to the two snapshots plotted. Left (right) snapshot during the cooling (warming) phase of the temperature, salinity and meridional overturning circulation are close to the thermal (haline) steady state: strong stratification of the 500 (1000) first meters and northward (southward) surface flux. The solid, dashed and dotted lines respectively correspond to positive, negative and zero anomalies: CI is respectively $2^{\circ} \mathrm{C}, 0.5$ psu and $1 \mathrm{~Sv}$ from (left) 4 to $24^{\circ} \mathrm{C}, 35$ to 37 psu and 0 to $15 \mathrm{~Sv}$; (right) 4 to $25^{\circ} \mathrm{C}, 31$ to $37 \mathrm{psu}$ and -4 to $3 \mathrm{~Sv}$. 

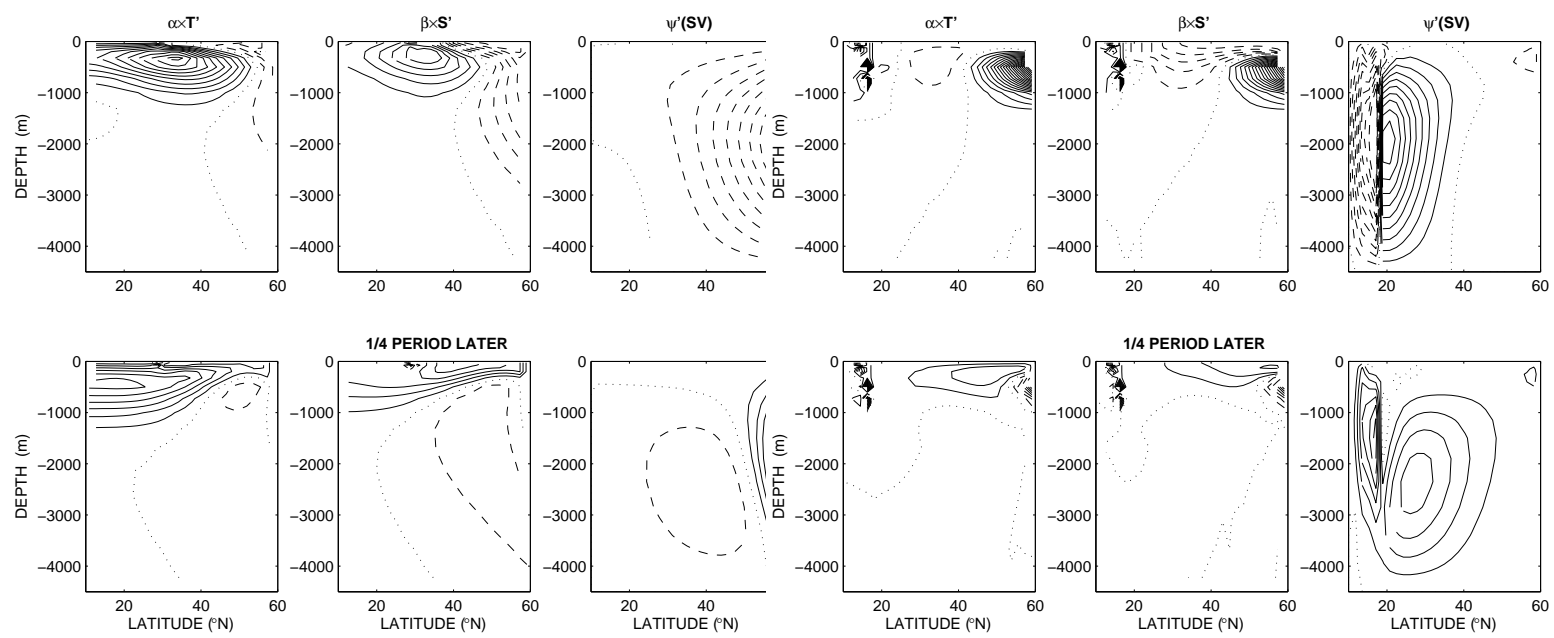

Figure 4: (left) Temperature, salinity (both in terms of density) and meridional overturning anomaly snapshots, of a phase and a quarter period later, of the oscillation occurring during the thermal bifurcation of the millennial oscillation. (right) As left for the limit cycle occurring close to the haline bifurcation. The period of this centennial oscillation are respectively 187 and $138 \mathrm{yr}$ for a freshwater flux intensity of 94 and $185 \mathrm{~m} \mathrm{yr}^{-1}$. The solid, dashed and dotted lines respectively correspond to positive, negative and zero anomalies: $\mathrm{CI}$ is respectively $5 \times 10^{-6}\left(5 \times 10^{-7}\right)$ and $0.5\left(10^{-2}\right) \mathrm{Sv}$. 

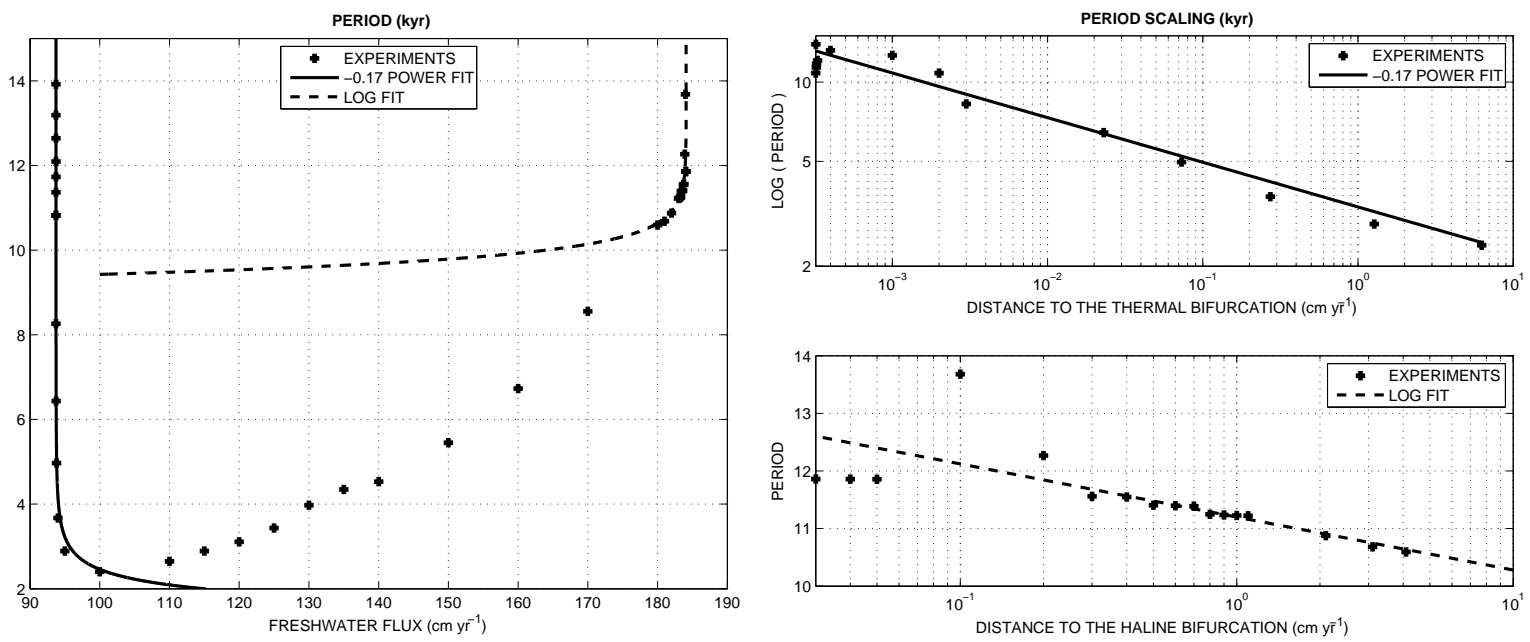

Figure 5: Millennial oscillation period as a function of the intensity of the freshwater flux: it becomes infinite near the two bifurcations, following an -0.17 power (logarithm) law between the regime of thermal (haline) steady state and of the millennial oscillation. 

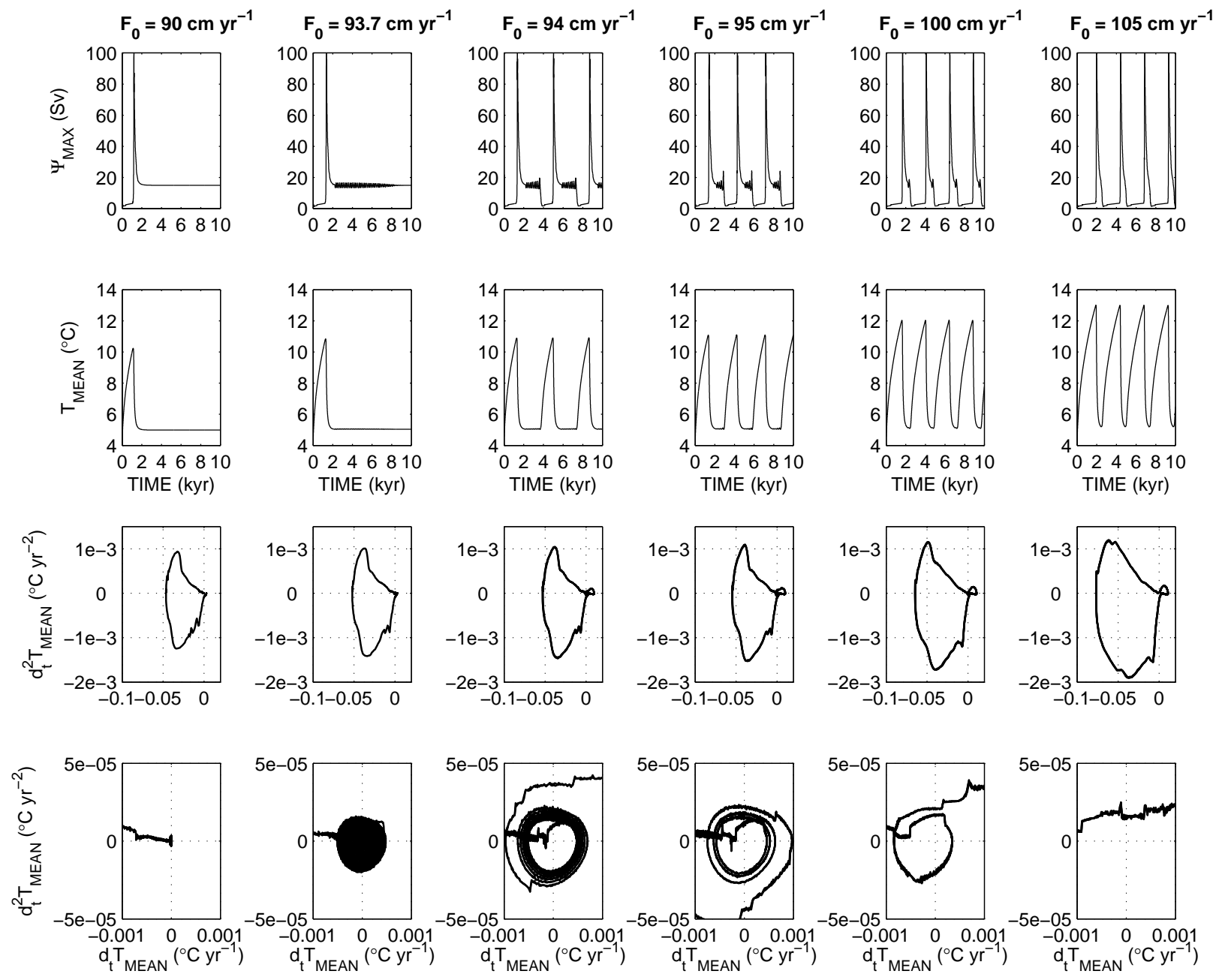

Figure 6: Time integration of the model for 6 different values of the freshwater flux around the first bifurcation. The maximum of the meridional overturning circulation (first row) and the mean temperature (second), show the appearance of the millennial oscillation and of higher frequency oscillation during the temperature steady state and phase. The phase portrait for the entire cycle (third row) and a blow-up around the zero value (fourth) show a stable spiral fixed point moving into an unstable spiral fixed points, then the trajectories follow the millennial stable cycle. The increasing freshwater flux increases the instability of the spiral fixed point. 

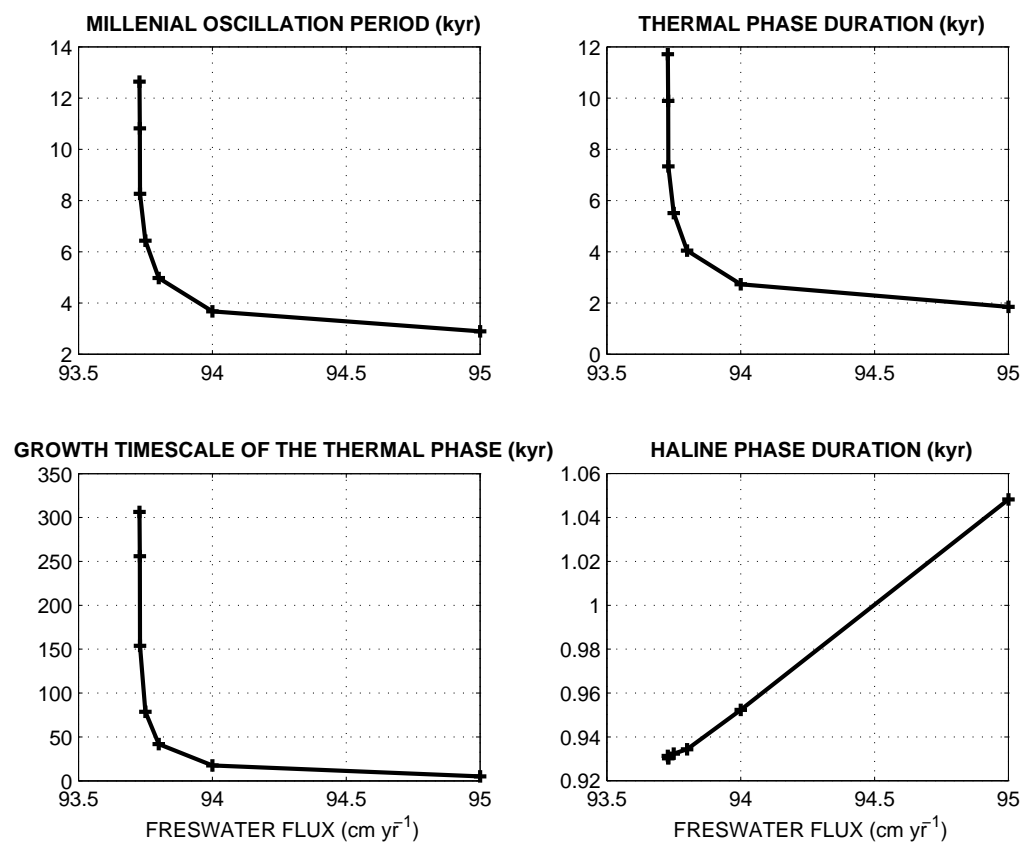

Figure 7: Millenial oscillations characteristics as a function of the freshwater flux amplitude: period (top left), time spent in the thermal (top right) and haline phase (bottom right) during a millennial oscillation, growth time scale of the thermal phase instability (bottom left). 

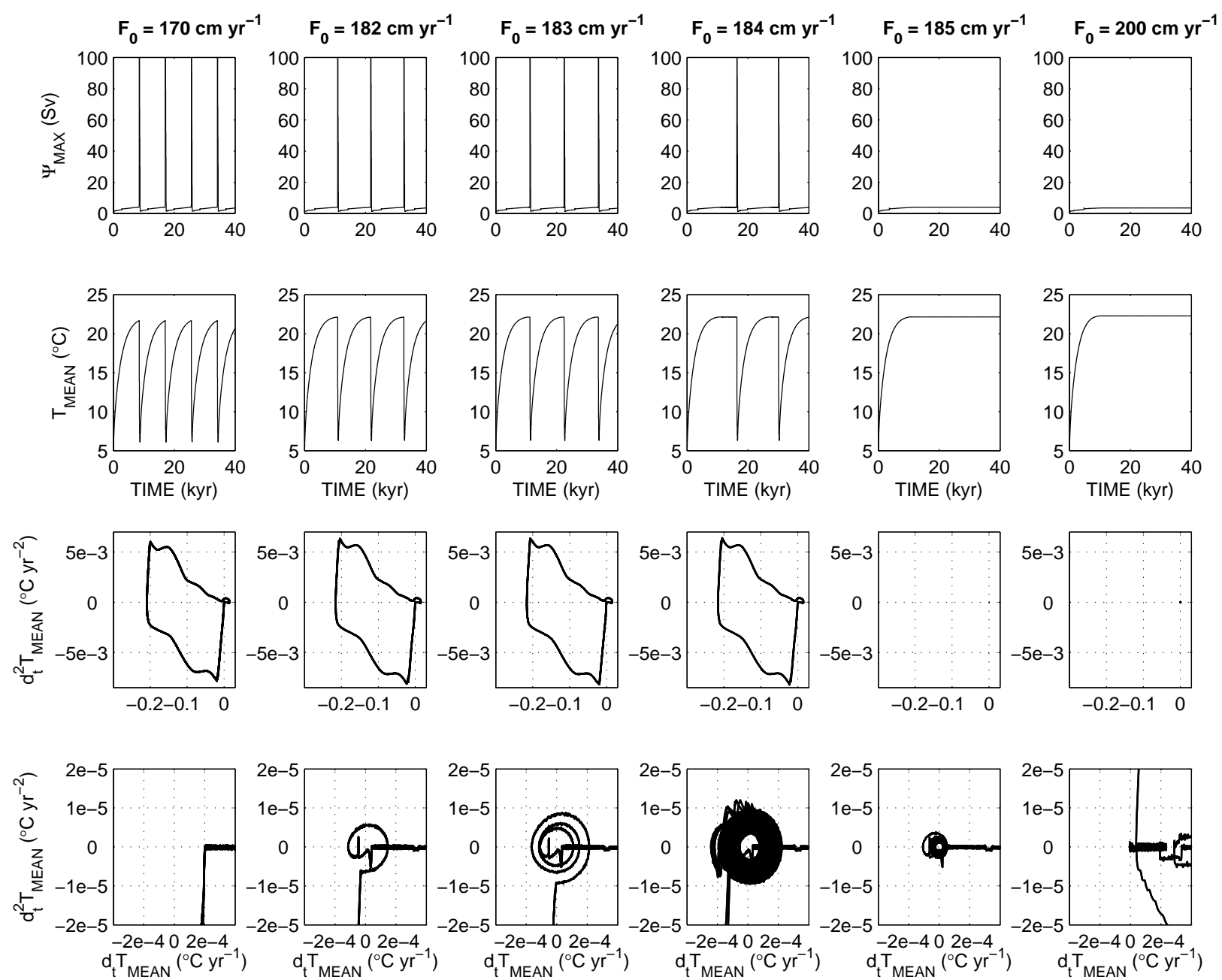

Figure 8: As in Fig. 6 for the second bifurcation. The diagrams show, trough the modification of the freshwater flux intensity, the appearance of an oscillation during the haline phase of the millennial cycle, then the appearance of a stable limit cycle which becomes weaker and weaker until it becomes a stable point (corresponding to the stable haline steady state). 

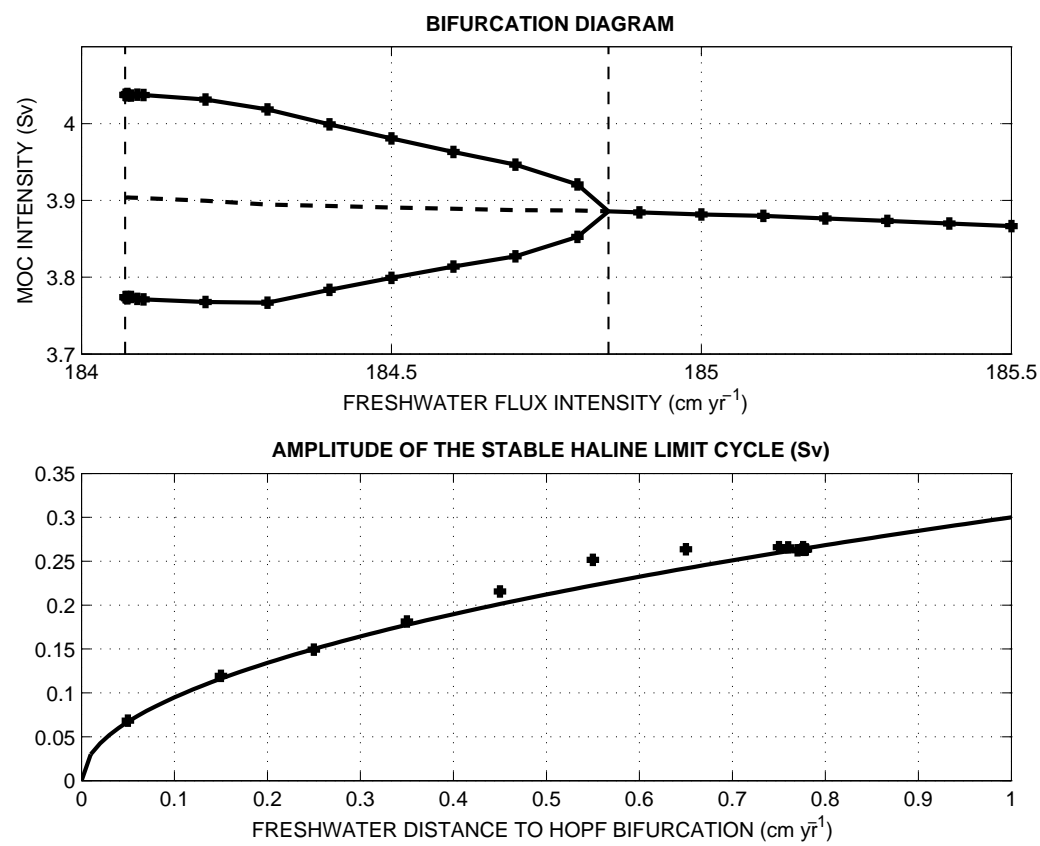

Figure 9: (top) As in Fig. 1, around the haline bifurcation. The dashed line represents the mean value of the limit cycle. (bottom) Amplitude of the stable limit cycle (+) as a function of the distance to the bifurcation, the square root law (solid line) characterizes the presence of a supercritical Hopf bifurcation. 

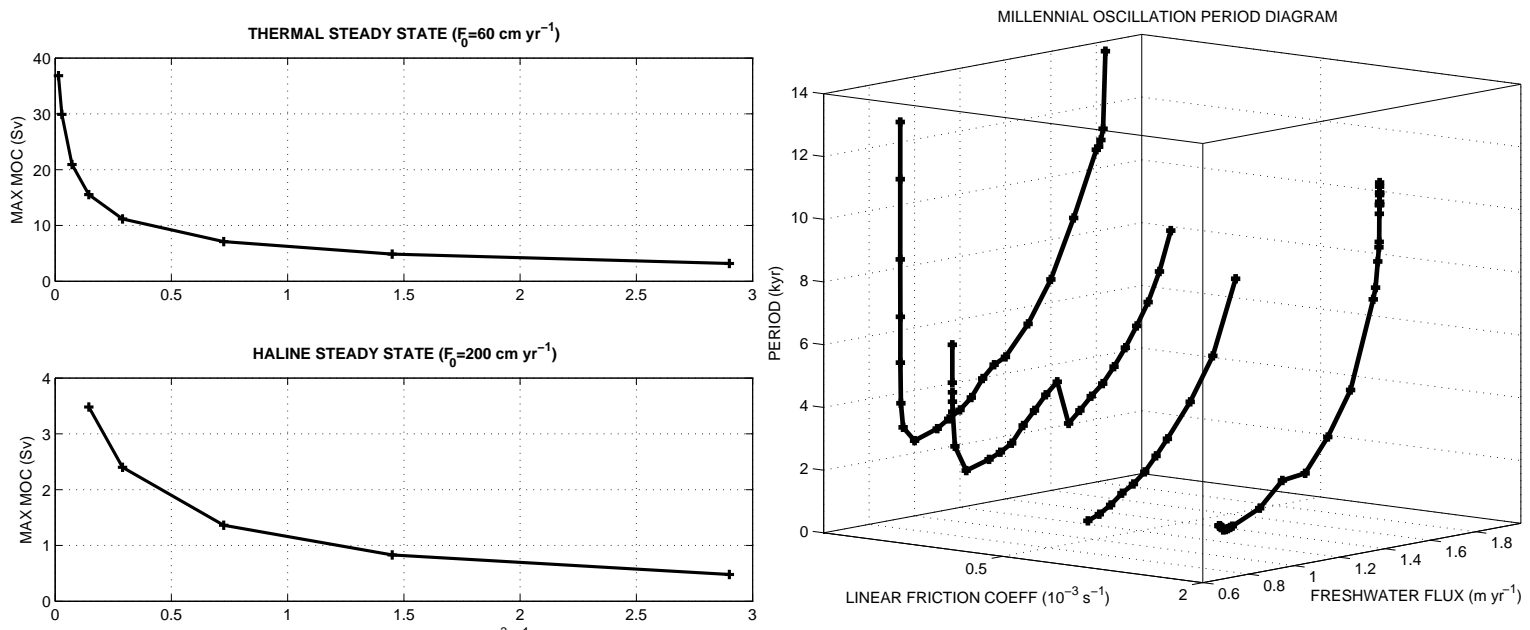

Figure 10: (right) Circulation intensity of the thermal and haline steady states through modification of the linear friction coefficient. (left) Millennial oscillation period as a function of freshwater flux intensity and linear friction coefficient. The first bifurcation behavior strongly depends of the linear friction coefficient: for the two series of experiments with the highest friction, the first bifurcation is no longer a bifurcation with an infinite period behavior. 


\section{List of Tables}

1 Parameters used for the 2D model time integrations. . . . . . . . . . 33 
Table 1: Parameters used for the 2D model time integrations.

\begin{tabular}{rll}
\hline$n_{y}$ & 28 & number of gridpoints in latitude \\
$n_{z}$ & 15 & number of gridpoints on the vertical \\
$H$ & $4500 \mathrm{~m}$ & ocean uniform depth \\
$W$ & $5120 \mathrm{~km}$ & zonal basin extent \\
$y_{0}$ & $10^{\circ} \mathrm{N}$ & southern boundary position \\
$y_{1}$ & $60^{\circ} \mathrm{N}$ & northern boundary position \\
$K_{H}$ & $10^{3} \mathrm{~m}^{2} \mathrm{~s}^{-1}$ & horizontal tracer diffusion \\
$K_{V}$ & $10^{-4} \mathrm{~m}^{2} \mathrm{~s}^{-1}$ & vertical tracer diffusion \\
$g$ & $9.8 \mathrm{~m} \mathrm{~s}^{-2}$ & gravity acceleration \\
$\rho_{0}$ & $1027 \mathrm{~kg} \mathrm{~m}^{-3}$ & reference density \\
$\alpha$ & $2.2 \times 10^{-4} \mathrm{~K}^{-1}$ & thermal expansion coefficient \\
$\beta$ & $7.7 \times 10^{-4} \mathrm{psu}^{-1}$ & haline contraction coefficient \\
$\tau_{T}$ & $66 \mathrm{days}^{-1}$ & temperature restoring time \\
$F_{0}$ & $60-200 \mathrm{~cm} \mathrm{yr}^{-1}$ & freshwater flux intensity \\
$T_{0}^{*}$ & $13.5^{\circ} \mathrm{C}$ & restoring temperature amplitude \\
$\tilde{y}$ & $76^{\circ}$ & restoring temperature extent \\
$\varepsilon$ & $1.45 \times 10^{-4} \mathrm{~s}^{-1}$ & linear friction coefficient \\
$\varepsilon$ & $2.90,7.25,18.49 \times 10^{-4} \mathrm{~s}^{-1}$ & other linear friction coefficients \\
\hline
\end{tabular}

